\title{
Visualizing the ac magnetic susceptibility of superconducting films via magneto-optical imaging
}

\author{
M. Motta, ${ }^{1}$ F. Colauto, ${ }^{1}$ R. Zadorosny, ${ }^{1, *}$ T. H. Johansen,,${ }^{2,3}$ R. B. Dinner, ${ }^{4}$ M. G. Blamire,${ }^{4}$ G. W. Ataklti, ${ }^{5}$ \\ V. V. Moshchalkov, ${ }^{5}$ A. V. Silhanek, ${ }^{5,6}$ and W. A. Ortiz ${ }^{1,3}$ \\ ${ }^{1}$ Departamento de Física, Universidade Federal de São Carlos, 13565-905 São Carlos, SP, Brazil \\ ${ }^{2}$ Department of Physics, University of Oslo, POB 1048, Blindern, NO-0316 Oslo, Norway \\ ${ }^{3}$ Centre for Advanced Study, Norwegian Academy of Science and Letters, NO-0271 Oslo, Norway \\ ${ }^{4}$ Department of Materials Science, University of Cambridge, Pembroke Street, Cambridge CB2 3QZ, UK \\ ${ }^{5}$ INPAC - Institute for Nanoscale Physics and Chemistry, Nanoscale Superconductivity and Magnetism Group, \\ Katholieke Universiteit Leuven, Celestijnenlaan 200D, B-3001 Leuven, Belgium \\ ${ }^{6}$ Département de Physique, Université de Liège, B-4000 Sart Tilman, Belgium
}

(Received 8 February 2011; revised manuscript received 4 October 2011; published 30 December 2011)

\begin{abstract}
We have established a link between the global ac response and the local flux distribution of superconducting films by combining magnetic ac susceptibility, dc magnetization, and magneto-optical measurements. The investigated samples are three $\mathrm{Nb}$ films: a plain specimen, used as a reference sample, and other two films patterned with square arrays of antidots. At low temperatures and small ac amplitudes of the excitation field, the Meissner screening prevents penetration of flux into the sample. Above a certain ac drive threshold, flux avalanches are triggered during the first cycle of the ac excitation. The subsequent periodic removal, inversion, and rise of flux occurs essentially through the already-created dendrites, giving rise to an ac susceptibility signal weakly dependent on the applied field. The intradendrite flux oscillation is followed, at higher values of the excitation field, by a more drastic process consisting of creation of new dendrites and antidendrites. In this more invasive regime, the ac susceptibility shows a clear field dependence. At higher temperatures a smooth penetration occurs, and the flux profile is characteristic of a critical state. We have also shown that the regime dominated by vortex avalanches can be reliably identified by ac susceptibility measurements.
\end{abstract}

DOI: 10.1103/PhysRevB.84.214529 PACS number(s): 74.78.Na, 74.78.Fk, 74.25.Dw, 74.25.Op

\section{INTRODUCTION}

Superconductivity is a state of matter characterized by a diamagnetic response caused by the exclusion of magnetic flux from its interior. In type II superconductors, the resulting field-dependent magnetic moment can be linked to the internal distribution of the quantum units of flux, so-called vortices. Indeed, a picture of the flux distribution at microscopic scales can be indirectly achieved from integrated response techniques, via the assumption of certain predetermined models. Arguably, the most successful and popular mapping between the microscopic and macroscopic superconducting worlds is the Bean critical state model. ${ }^{1,2}$ This model allows one to estimate, from magnetization and susceptibility measurements, the maximum current a superconductor can bear before dissipating, i.e., its critical current. The basic assumptions validating the Bean model are the existence of a continuous flux distribution throughout the specimen and a field-independent critical current. Clearly, such restrictive conditions do not account for all possible scenarios of flux distribution. Attempts for adjusting to more realistic situations included the decrement of critical current with magnetic field ${ }^{3}$ the presence of edge barriers, ${ }^{4,5}$ and the existence of a finite lower critical field. ${ }^{6,7}$ In most cases, these improvements result in marginal corrections to the simpler Bean approximation.

A situation in which the Bean critical state model fails dramatically occurs when sudden flux bursts develop as a consequence of a thermomagnetic instability. ${ }^{8}$ Under these circumstances, any estimation of the critical current based on global response techniques is futile and local magnetic probes become imperative. An unambiguous identification of the field range dominated by avalanches can be obtained through the field dependence of the magnetization, which exhibits clear jumps within the instability region. ${ }^{9-11}$ However, signatures of the avalanche regime in the ac susceptibility are not easy to recognize, most specially for values of the ac drive $h$ sufficiently small to avoid disturbance on the flux distribution which one intends to probe. For larger values of $h$, however, both components of the ac susceptibility, $\chi=\chi^{\prime}+j \chi^{\prime \prime}$, are reentrant in temperature and applied field; i.e., shielding becomes stronger and dissipation decreases upon increase of either of those two variables. This reentrant behavior has also been observed earlier in $\mathrm{Pb}$ films ${ }^{12,13}$ and ascribed to the occurrence of flux avalanches. The present work reveals the cause for such change of the diamagnetic response through investigations of the early stages of flux penetration in $\mathrm{Nb}$ films. In our approach we performed magnetic measurements employing global techniques-namely, susceptibility and magnetization - as well as magneto-optical imaging (MOI). Our experiments revealed that the first avalanches, while serving as a track for flux entrance, do also guide flux exit, thus suggesting why the initial ac response of the superconducting film is nearly constant for variations of the applied field $H$ or temperature $T$. Upon increase on $H$ or $T$, the ac response reenters, i.e., becomes more diamagnetic as the avalanches cease.

\section{SAMPLES AND EXPERIMENTAL TECHNIQUES}

The samples investigated consist of $\mathrm{Nb}$ films of approximately rectangular shape, with thickness $d=50 \mathrm{~nm}$. Their lateral dimensions appear in Table I. Two of them have a square array of antidots (ADs) fabricated by electron-beam lithography. The $\mathrm{Nb}$ was deposited via dc sputtering on top 
TABLE I. Superconducting critical temperature $T_{c}$, coherence length $\xi_{\mathrm{GL}}(0)$, penetration depth $\lambda_{\mathrm{GL}}(0)$, and lateral dimensions $l$ and $w$ for the three samples investigated.

\begin{tabular}{lccccc}
\hline \hline Sample & $T_{c}(\mathrm{~K})$ & $\xi_{\mathrm{GL}}(0)(\mathrm{nm})$ & $\lambda_{\mathrm{GL}}(0)(\mathrm{nm})$ & $l(\mathrm{~mm})$ & $w(\mathrm{~mm})$ \\
\hline Pristine & 7.91 & 9.4 & 168.0 & 3.0 & 2.5 \\
AD04 & 7.40 & 9.1 & 170.0 & 2.7 & 2.6 \\
AD08 & 6.42 & 9.1 & 173.0 & 2.7 & 2.5 \\
\hline \hline
\end{tabular}

of a $\mathrm{SiO}_{2}$ insulating substrate. Our preliminary investigations included a variety of similar samples, all of them leading to comparable results. The present report, however, is restricted to results on only three samples, one of each type. Thus, the sample investigated through magnetic measurements is the very same which was imaged using the MO technique. The patterned samples-named AD04 and AD08-have antidots with sizes $0.4 \mu \mathrm{m}$ and $0.8 \mu \mathrm{m}$, respectively. The third specimen - the reference sample - is a plain film of $\mathrm{Nb}$. The period of the patterns is $a=1.5 \mu \mathrm{m}$, which corresponds to a commensurability field $H_{1}=\frac{\Phi_{0}}{a^{2}}=9.2$ Oe at which the densities of vortices and antidots match each other. ${ }^{14}$ Using the expression for the upper critical field and for the dirty limit, ${ }^{15}$ the zero-temperature superconducting coherence length, $\xi_{\mathrm{GL}}(0)$, and the penetration depth, $\lambda_{\mathrm{GL}}(0)$, were estimated. The obtained values are shown in Table I, as well as the superconducting transition temperature at zero field, $T_{c}$. Noticeably, a drop in $T_{c}$ results from the insertion of antidots.

Measurements were made for temperatures ranging from $2 \mathrm{~K}$ up to $T_{c}$, and the ac sinusoidal drive was simulated, in MOI experiments, by cycling the dc field in complete loops made up of 0.1 Oe steps. Ac susceptibility and dc magnetization measurements were carried out with commercial Quantum Design equipment (MPMS and PPMS). The susceptibility measurements were recorded using a fixed frequency of $1 \mathrm{kHz}$, for values of the excitation field within the \pm 15 Oe interval. Prior to fixing the value of the excitation frequency, we verified that the susceptibility response is independent of frequency within 5 orders of magnitude, up to values above $1 \mathrm{kHz}$. In all cases, the excitation field $h$ and the external magnetic field $H$ were applied perpendicular to the film plane. The magneto-optical imaging technique employed relies on the occurrence of the Faraday effect ${ }^{16}$ on a magnetic indicator film placed on top of the superconducting specimen. The indicators used in the present work are Bi-substituted yttrium iron garnet films (Bi:YIG) with in-plane magnetization. Data for the so-called Cole-Cole plots of the susceptibility, i.e., its imaginary part $\chi^{\prime \prime}$ as a function of the real part $\chi^{\prime}$, were taken isothermally, varying the ac drive. Data normalization of the susceptibility was performed using the reference value $\chi_{0}$ of its real component, which represents the Meissner plateau, measured in the PPMS at $T=2 \mathrm{~K}$ with a drive amplitude $h=$ $20 \mathrm{mOe}$, in the absence of magnetic field. ${ }^{17}$

\section{RESULTS AND DISCUSSION}

It is well known that thin films of most superconducting materials exhibit flux avalanches within specific intervals of temperature and applied field. ${ }^{9-11,18-25}$ Such behavior is intimately correlated to thermomagnetic instabilities experienced by the material when heat generated by a sudden displacement of vortices cannot be dissipated, creating thus an increase in the local temperature. This warmer region, in turn, has its pinning capability reduced, being thus likely to host even more vortex motion, reinforcing the process. ${ }^{26,27}$ There is a threshold temperature $T_{\text {th }}$ above which no avalanches occur and a rather smooth penetration of the magnetic flux is observed. It has been shown that $T_{\text {th }}$ can be substantially increased if antidots are introduced into the specimen. ${ }^{9,12,13,28,29}$ In this case, the most relevant difference, compared to plain films, is that vortices are guided preferentially along the rows of ADs. ${ }^{13,29}$ This aspect will be further discussed later in this paper. Above $T_{\mathrm{th}}$, flux invades the sample gradually, and the system appears to obey a critical-state regime. Assuming the simplest of such critical-state regimes, i.e., the Bean model, one can obtain the superconducting critical current density $J_{c}$ by measuring the field $h$ at which the magnetic flux front reaches a characteristic geometry-dependent penetration distance. ${ }^{30,31}$ If one considers a thin disk of thickness $d$ and diameter $2 R \gg d$, the two-dimensional effective penetration depth in the perpendicular geometry is $\Lambda=\frac{2 \lambda^{2}}{d}$. For $\Lambda \ll 2 R$, the field amplitude at which $\chi^{\prime \prime}$ peaks, $h_{p}$, corresponds to $72 \%$ of the full penetration field and can be related to the critical current density by the expression $J_{c}=\frac{1.03 h_{p}}{d} .{ }^{32}$ On the other hand, an approximate expression for $J_{c}$ has been recently developed by Chen and co-authors ${ }^{33}$ for films of rectangular shape. We have employed Eq. (20) of Ref. 33 to calculate $J_{c}$ of our rectangular samples:

$$
J_{c}=\frac{4.85 \Upsilon_{0}(\gamma)}{\left(3-\gamma^{-1}\right) w} h_{p},
$$

where $\gamma=l / w, \tau=d / w$, and

$$
\Upsilon_{0}(\gamma)=\frac{\arctan \left(1-0.7223 \gamma^{-0.954}+0.3522 \gamma^{-2.57}-0.141 \gamma^{-3.66}\right)}{\tau} .
$$

Figure 1 compiles results obtained from isothermal ac susceptibility measurements on the three films, taken with the PPMS in zero applied field, ${ }^{17}$ using the excitation amplitude $h \leqslant 15$ Oe as the external variable. Panel (a) shows $J_{c}\left(\frac{T}{T_{c}}\right)$ obtained as discussed in the previous paragraph, with $h_{p}$ determined from the peak in $\chi^{\prime \prime}(h)$ curves. We have used the reduced variable $t=\frac{T}{T_{c}}$ to unify the superconducting domain of all studied samples. Curves for the plain film and sample AD04 have two distinct regions: At higher temperatures, $J_{c}(T)$ is smooth, with upward curvature; below 

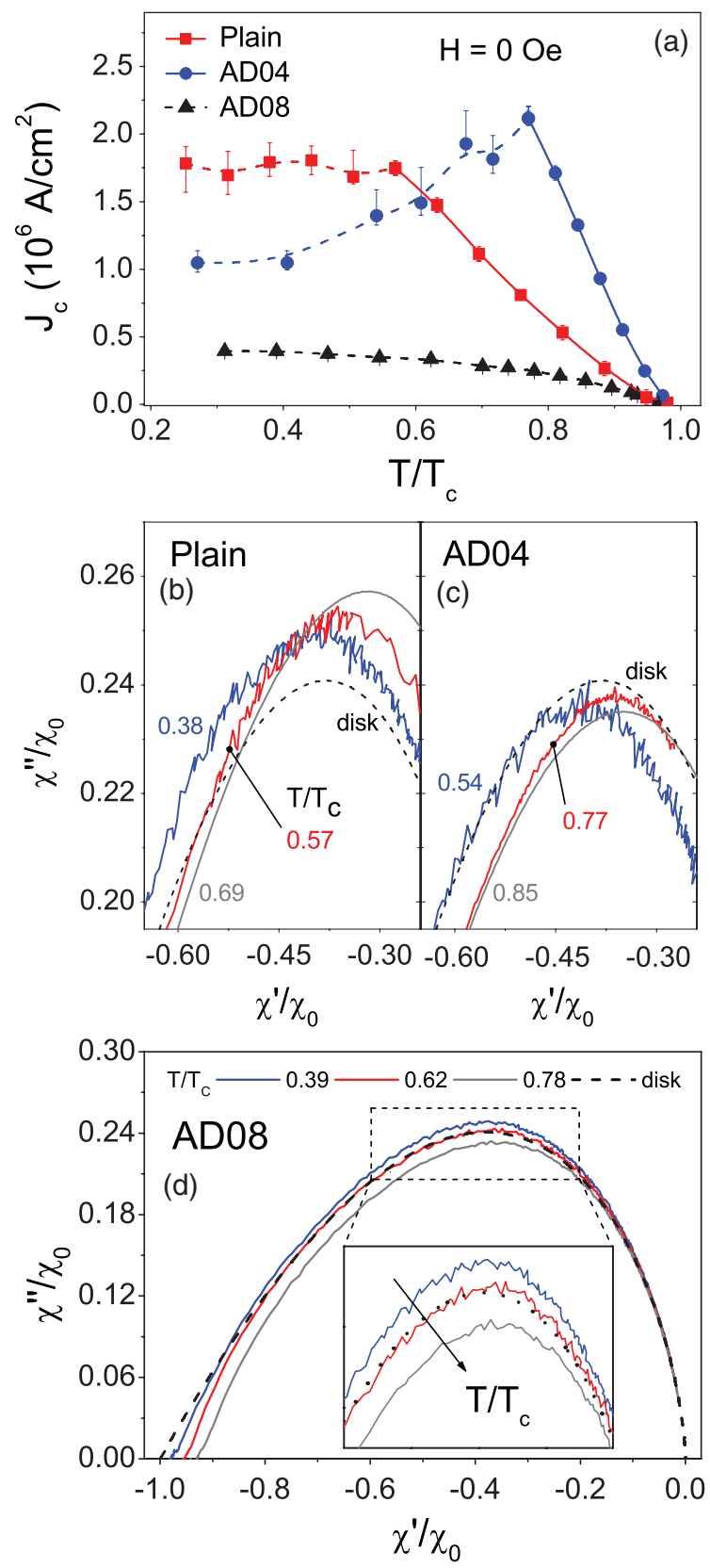

FIG. 1. (Color online) Panel (a): Temperature dependence of the critical current in the absence of a dc field (see main text and Ref. 17), as determined from the peak on the imaginary part of the susceptibility measured as a function of the excitation amplitude. Panels (b)(d): Cole-Cole plots, at different temperatures, for the studied samples: pristine (b), AD04 (c), and AD08 (d). The theoretical plot for a thin circular disk is also shown. Smoothness or noisiness of the Cole-Cole plots emphasizes different regimes (critical state or avalanches) of flux penetration.

a certain limiting temperature, however, it deviates from this canonical behavior, a change that is intimately connected to the appearance of avalanches in both specimens, as explained later in this paper. The equivalent curve for sample AD08 is quite different: A downward curvature is maintained throughout the whole interval, as a consequence of avalanches being present within the entire temperature domain. As a matter of fact, values of $J_{c}$ shown for temperatures below the avalanche threshold should not be interpreted as representative of the critical current density, since they were obtained under the erroneous assumption that the system is in a critical-state regime. In the avalanche regime, the actual critical current is a local, extremely inhomogeneous variable, which cannot be properly described by a unique global value. Nonetheless, displaying all points in a single picture stresses the idea that a frontier between both regimes can be reliably obtained from ac susceptibility measurements. It should be noticed that the curves in Fig. 1(a) confirm that the inclusion of ADs of moderate size promotes an increase in $J_{c}{ }^{34}$ and $T_{\mathrm{th}} \cdot{ }^{9,13}$ Comparing the high- $T$ portions of $J_{c}(T)$ for the plain film and sample AD04, one clearly sees this improvement, as well as the relative enlargement of the area where avalanches occur. For sample AD08, however, this comparison does not apply, since avalanches take place in the whole temperature window.

A second important signature of the occurrence of avalanches that can be drawn from susceptibility measurements is depicted in panels (b) through (d) of Fig. 1, which show Cole-Cole plots of the susceptibility. Displaying data in such a manner can be quite advantageous to emphasize the occurrence of different regimes of vortex matter. For example, all Cole-Cole plots for a system obeying a critical state dynamics should collapse into one single universal plot, irrespective of the values of applied field, temperature, frequency, and amplitude of the ac drive. Panel (b) shows results around the peak for the pristine film, taken at three different values of $T$ : For temperatures below the threshold limit for avalanches ( $t=0.38$ and 0.57$), \chi^{\prime \prime}\left(\chi^{\prime}\right)$ is very noisy, contrasting with the behavior observed at higher temperatures $(t=0.69)$, for which the curves are noticeably smooth. A similar set of curves is shown in panel (c) for sample AD04, which exhibits avalanches for $t=0.54$ and $t=0.77$, but not for $t=0.85$. Complete Cole-Cole plots for sample AD08 are shown in panel (d) for $t=0.39, t=0.62$, and $t=0.78$. The inset features the central portion of the curves, emphasizing the noisy behavior which, for this sample, is present over the entire range of temperatures. It should be noticed that, at the left lower part of Fig. 1(d), $\chi^{\prime \prime}$ goes to zero while $\chi^{\prime}$ is not -1 for larger values of $T$. The reason for this behavior is that the initial screening on curves $\chi^{\prime}(h)$ remains flat but imperfect, while there is essentially no dissipation $\left[\chi^{\prime \prime}(h) \sim 0\right]$. This means that some flux enters but remains pinned. Since this behavior is also exhibited by sample AD08 and the pristine film, one is led to associate it with the intrinsic pinning of $\mathrm{Nb}$. We will return to this point further ahead.

It is also worth comparing the experimental results with those expected theoretically. Chen et al. ${ }^{33}$ have shown that the ac susceptibility of rectangular films can be adequately treated using the same expression derived by Clem and Sanchez for an infinitely thin circular disk. ${ }^{32}$ This result, also included in all Cole-Cole plots of Fig. 1, indicates that, at all temperatures, the plain-film response [panel (b)] is different from that obtained for the Bean approximation, a result that is possibly due to the fact that $J_{c}$ is not field independent, as assumed in that simple model. As a matter of fact, the Bean model predicts a peak at $\chi_{\max }^{\prime}=0.38$, whereas the assumption of a field dependence on $J_{c}$ shifts the peak to the right and up, ${ }^{35}$ just as observed for the pristine film. On the other hand, the Cole-Cole plots for the 

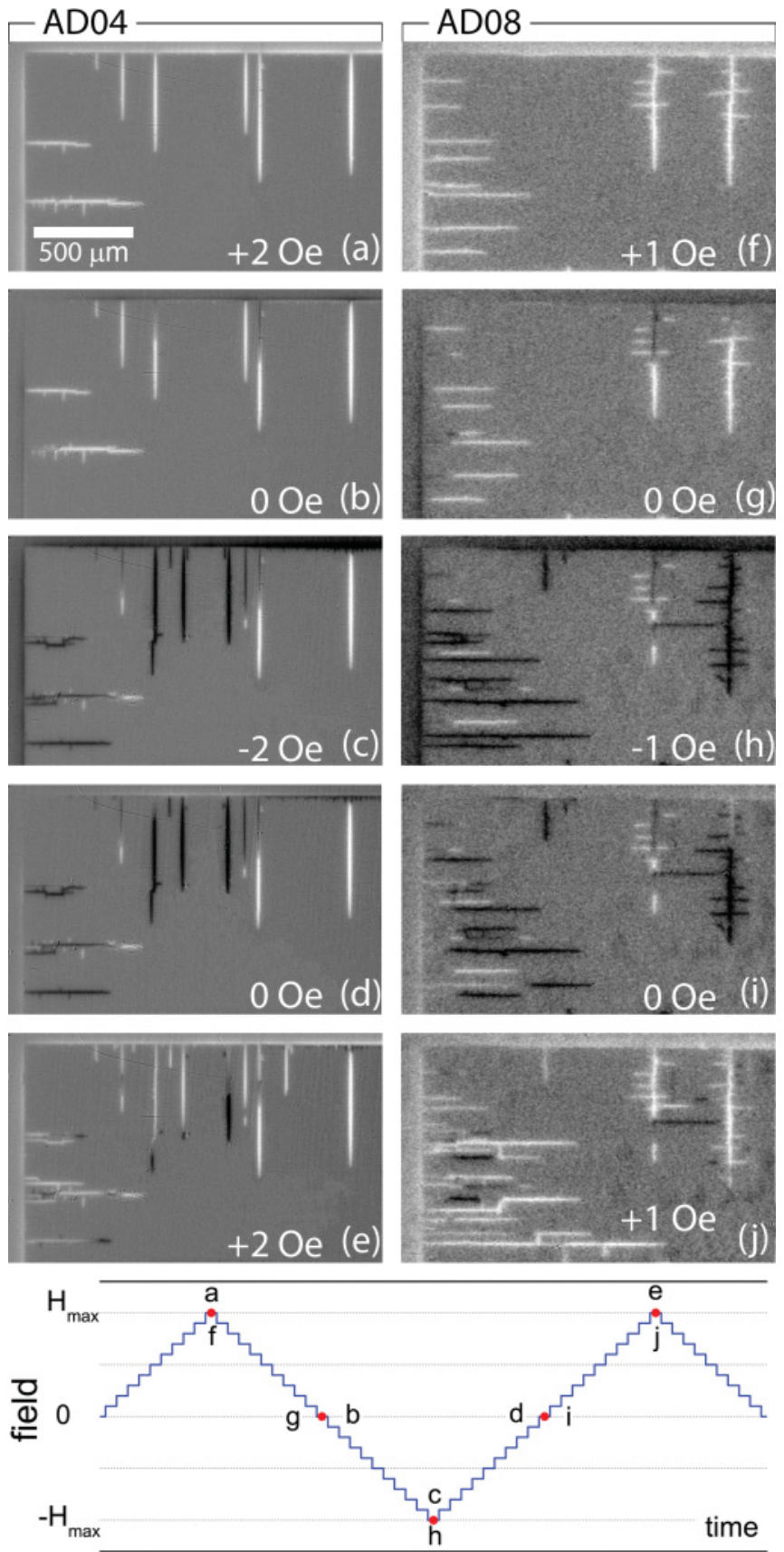

FIG. 2. (Color online) MO images taken at $3 \mathrm{~K}$. White indicates positive flux, dark negative, medium gray stands for zero flux (screened). Panels (a) to (e) are for sample AD04: After zero-field cooling, field increased to 2 Oe (a), decreased to zero (b) and -2 Oe (c), and increased back to zero (d) and 2 Oe (e). Same protocol followed for sample AD08, panels (f) to (j), with field extremes of \pm 1 Oe. Bottom panel: Sketch of time evolution of the applied field during data collection.

patterned samples deviate from the one predicted by Bean's model in a nonsystematic manner, so that the difference cannot be ascribed to a specific mechanism-e.g., the introduction of creep or of a field-dependent $J_{c}{ }^{35-37}$

We turn now the discussion to the images of profiles of flux penetration and exit at relatively small fields. Such images where taken after cyclic field excursions, as in the case

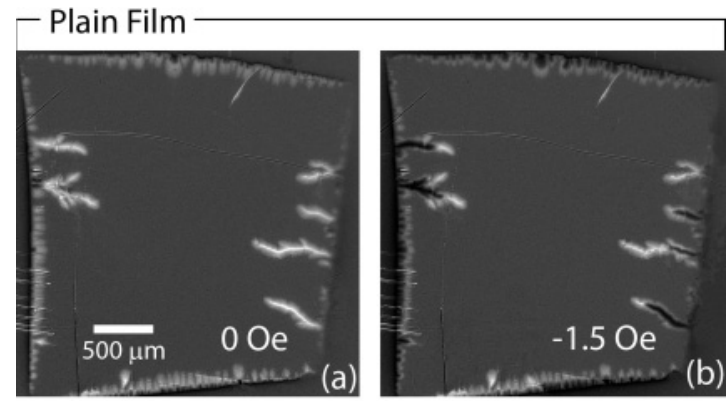

FIG. 3. MO images of the pristine film, taken at $3 \mathrm{~K}$, following the same protocol depicted in Fig. 2: After zero-field cooling, field was cycled between \pm 4 Oe. Panel (a) was taken at 0 Oe after the first half cycle of the field; panel (b) is for -1.5 Oe.

of those performed during susceptibility measurements. To emulate the magnetic history imprinted by the ac excitation, the external field was ramped up and down in steps of 0.1 Oe. However, given the time needed to acquire images, on the order of $100 \mathrm{~ms}$, the cycles were not sinusoidal, but steplike, as roughly indicated at the bottom of Fig. 2. The similarity among this procedure and that taking place during a sinusoidal cycle in a susceptibility measurement is ensured by the frequency independence of the ac measurements. Figures 2 and 3 present some selected MO images for the three samples studied here. All pictures were taken for $T=3 \mathrm{~K}$. White regions correspond to positive penetrated flux, dark areas represent negative flux (antiflux), while medium gray stands for unpenetrated portions of the sample (zero flux). Panels (a) to (e) in Fig. 2 are for sample AD04: After a zero-field cooling procedure, the field was increased to 2 Oe (a), decreased back to zero (b) and to -2 Oe (c), and then increased again to zero (d) and finally to $2 \mathrm{Oe}$ (e). All steps were performed with the ramp rate of $20 \mathrm{Oe} / \mathrm{s}$. We find that near $1.4 \mathrm{Oe}$, abrupt avalanches suddenly invade the sample from the edge, in the form of finger-type dendrites, clearly guided by the rows of ADs (a). Upon decrease of the field, reversed flux penetrates the sample through the same tracks [(b) and (c)], transforming them progressively into antidendrites. When the field is increased again $[(\mathrm{d})$ and (e)], the tracks are penetrated once more by positive flux, so that the original dendrites are gradually restored. The same behavior is seen in panels (f) to (j) for sample AD08, for which the field protocol is just the same as for AD04, with field extremes of \pm 1 Oe. This feature is also presented by the pristine sample, as exemplified in panels (a) and (b) of Fig. 3, taken respectively at fields zero and -1.5 Oe, after a field excursion to 4 Oe.

It is worth mentioning that all samples studied here exhibit dendritic penetration at certain values of field and temperature. However, for experiments repeated under the same conditions, flux dendrites follow different tracks during each run. This further emphasizes that the onset of flux avalanches is governed by an instability condition. The lattice of antidots is nevertheless causing some degree of guidance for the full avalanche, in contrast to the dendritic penetration in unpatterned samples.

We have also used magnetic measurements and MO imaging to investigate the reentrant behavior of susceptibility curves. The inset of Fig. 4(a) shows the temperature 

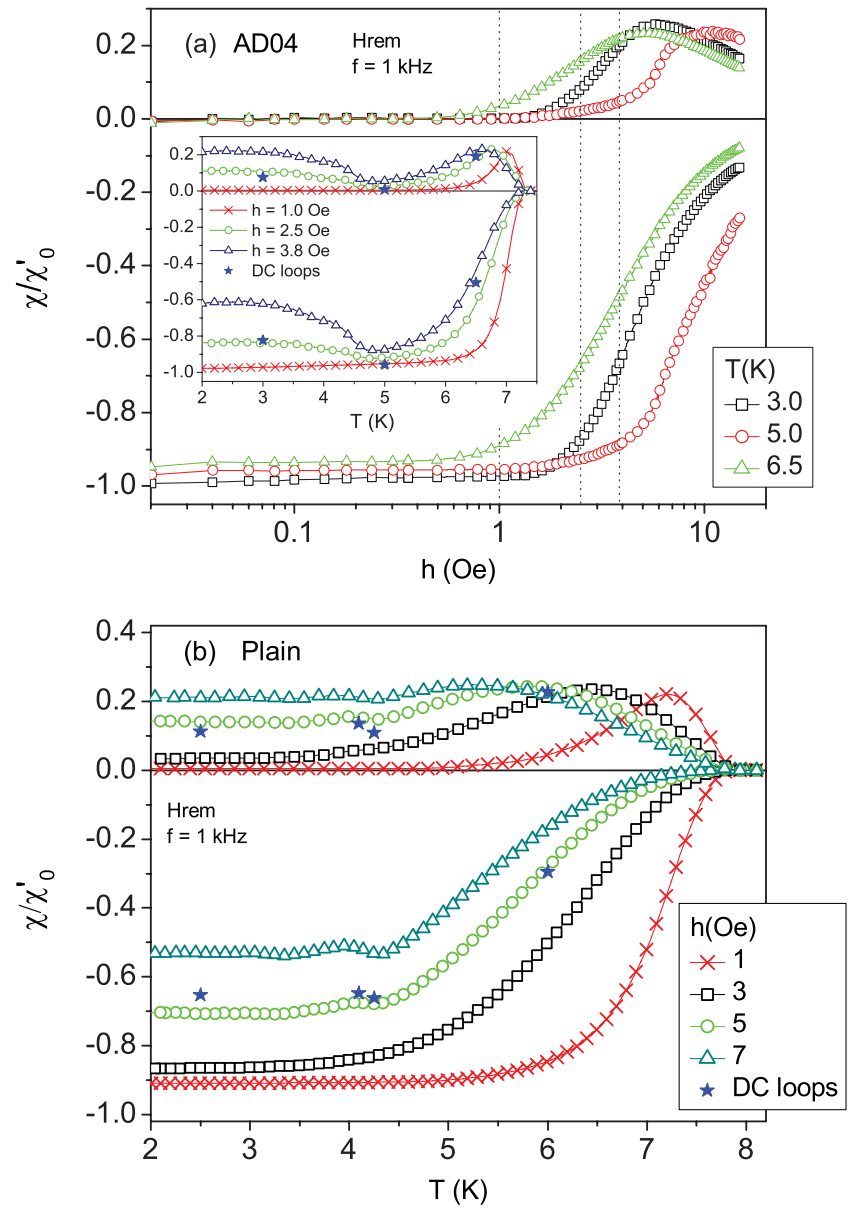

FIG. 4. (Color online) (a): Real and imaginary parts of $\chi$ as a function of the ac amplitude, taken at $T=3,5$, and $6.5 \mathrm{~K}$. The inset shows the temperature dependence of the same quantities, measured with $h=1,2.5$, and 3.8 Oe. Stars represent values of both components, at 3, 5, and $6.5 \mathrm{~K}$, calculated from magnetization loops (see text). (b): Similar to inset in (a) for the pristine film.

dependence of $\chi^{\prime}$ and $\chi^{\prime \prime}$, measured with ac amplitudes of 1.0, 2.5, and 3.8 Oe, for sample AD04. For the lower value of $h$, one sees the ordinary ac response of a superconductor; at larger amplitudes, however, the reentrant behavior appears, as already seen earlier in $\mathrm{Pb}$ films: ${ }^{12}$ Upon increase of the temperature, the real part first decreases, to a more diamagnetic level, and then increases toward zero at the transition. The imaginary part is also odd, starting at a relevant dissipation level and then decreasing before peaking as the transition is approached. One can also follow the evolution of both susceptibility components with the excitation field at fixed temperatures. The main graph of Fig. 4(a) shows isothermal measurements at 3,5 , and $6.5 \mathrm{~K}$. It is rather intriguing that the loss of diamagnetism starts earlier for $3 \mathrm{~K}$ than for $5 \mathrm{~K}$, a feature that is also matched by the peaks on $\chi^{\prime \prime}$. Further increasing $T$, however, restores the ordinary behavior; i.e., the transition is broadened and starts at lower amplitudes $h$. The vertical dashed lines on the main panel are guiding lines to connect both experiments: For $h=1 \mathrm{Oe}$, the real part $\chi^{\prime}$ increases monotonically as the temperature changes from 3 to 5 , and then to $6.5 \mathrm{~K}$. The imaginary part is also monotonous: negligible for 3 and $5 \mathrm{~K}$, and nonzero at $6.5 \mathrm{~K}$. On the other hand, for $h=2.5$ Oe both components have an initial decrease as $T$ is switched from 3 to $5 \mathrm{~K}$, followed by an increase, for $T$ growing from 5 to $6.5 \mathrm{~K}$. This feature is even more pronounced at large $h$. One can also notice that, for small values of $h, \chi^{\prime}(h) \sim-1$ at low temperatures (e.g., $3 \mathrm{~K}$ ), but is less negative for larger values of $T$, whereas the corresponding $\chi^{\prime \prime}(h) \sim 0$ in all temperatures. As discussed earlier, this is due to efficient pinning: Flux enters the sample but is prevented from moving. For this reason, the left-lower parts of the Cole-Cole plots for all samples studied here do not collapse, as seen, for example, in Fig. 1(d) for sample AD08. This reentrant behavior, discussed here for sample AD04, is also presented by the pristine film, as illustrated in Fig. 4(b) for the temperature dependence of $\chi^{\prime}$ and $\chi^{\prime \prime}$ for a variety of values of the excitation amplitude. However, as sample AD08 exhibits avalanches in the whole temperature interval, there is no transition between different dynamic regimes, and therefore no reentrance could occur, as is actually the case.

Invoking frequency independence of the susceptibility, one can emulate an ac measurement by cycling the dc field and capturing images of the penetration profile of the sample at adequate values of $H$. Panels (a)-(c) on Fig. 5 show hysteresis loops for sample AD04 at temperatures $3 \mathrm{~K}, 5 \mathrm{~K}$, and $6.5 \mathrm{~K}$, respectively. At $3 \mathrm{~K}$ one sees a hysteretic loop whose nonvanishing area is due to the viscous motion of entering and exiting vortices within the AD-guided dendrites [panel (d)]. As shown on panel (c), at $6.5 \mathrm{~K}$ the loop is wide open, as could be expected for temperatures approaching $T_{c}$. The corresponding image is shown on panel (f), with a critical-state-like envelope and a certain "microtexture." Flux entrance is not abrupt, as in an avalanche, exhibiting a filamentary though smooth penetration. Mostly interesting, however, is the fact that the magnetic response at $5 \mathrm{~K}$ is nonhysteretic, ${ }^{38}$ which means

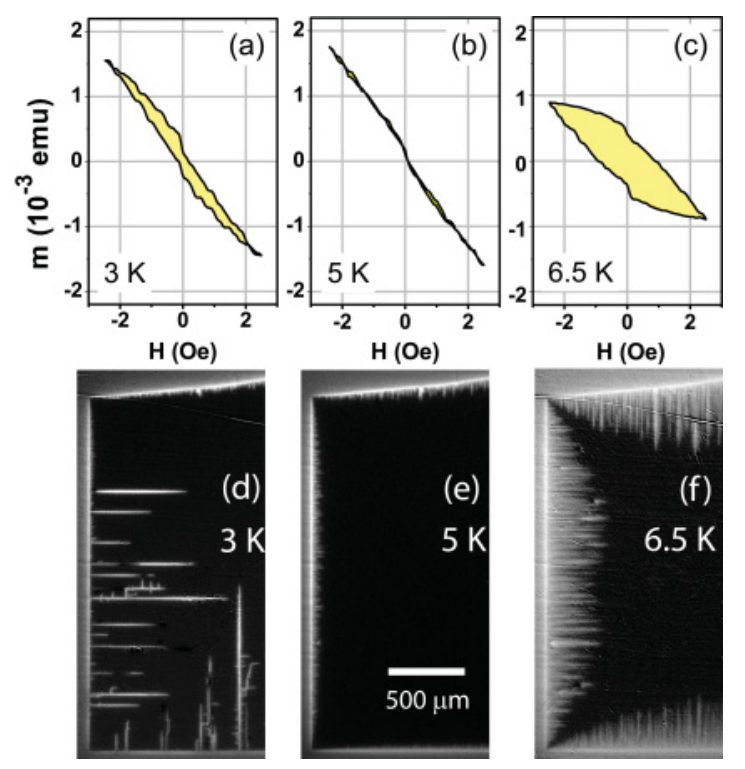

FIG. 5. (Color online) Panels (a)-(c): Hysteresis loops for sample AD04 at temperatures 3, 5, and 6.5 K. The area of the loop, which is nonzero at $3 \mathrm{~K}$, closes down at $5 \mathrm{~K}$ and reopens at $6.5 \mathrm{~K}$. Panels (d)-(f) are the corresponding images at the maximum field (2.5 Oe), taken immediately after the virgin curve. 
that, upon increase of the temperature, the loop first closes down and then opens up again. The MO image, represented on panel (e), shows quite clearly that the heart of the sample is not penetrated by magnetic flux at $5 \mathrm{~K}$ and $2.5 \mathrm{Oe}$. We have also compared hysteresis loops and MO images for the pristine sample, obtaining similar results. The collapse of the loop, however, does not occur for sample AD08 which, as already discussed, always exhibits avalanches. We take this as an additional evidence that the closing down of the loop is a feature intimately related to the suppression of avalanches in samples AD04 and pristine.

One further evidence that ac measurements can be emulated using dc magnetization loops arises from the argument that $\chi^{\prime}$ is the average slope of the magnetization loop (throughout one full cycle), whereas $\chi^{\prime \prime}$ is related to the energy losses per sample volume per cycle by

$$
\chi^{\prime \prime}=\frac{\oint M d H}{\pi H_{m}^{2}}=\frac{A}{\pi H_{m}^{2}},
$$

where $A$ is the area of the magnetization hysteresis loop, which extends from $-H_{m}$ to $+H_{m} \cdot{ }^{32,39}$ Employing this reasoning, we calculated both components of $\chi$ using data from the dc loops. As an illustration we have included, in the inset of Fig. 4(a), stars representing such results as obtained from the $\pm 2.5 \mathrm{Oe}$ loops measured at 3,5 , and $6.5 \mathrm{~K}$. A similar procedure for the pristine film, taken with $H_{m}=5 \mathrm{Oe}$, leads to the set of stars on Fig. 4(b). One can thus conclude that the reentrant behavior of $\chi^{\prime}$ - i.e., a reinforced diamagnetism - is related to the temperature limit for the occurrence of avalanches. ${ }^{10,11}$

As a final remark, we comment on the filamentary structure of the penetrated flux on sample AD04 at high temperatures. Panel (f) in Fig. 5 shows this clearly: While the penetration front has the typical format of a critical-state regime, it is in fact an envelope for a filamentary finger-type structure. Noticeably, however, no avalanches take place at that temperature, and the filamentary inner structure develops smoothly. From time to time, the smooth penetration is perturbed by small amounts of flux entering the film. Minor fluctuations seen on $M(H)$ at $6.5 \mathrm{~K}$ [Fig. 5(c)] are the corresponding signatures of those tiny perturbations. This interesting feature, which is in straight connection with the existence of the array of ADs, can be compared with another occurrence of a filamentary structure, observed by Welling et al. in a YBCO film with an array of ADs. ${ }^{40}$ Flux penetration in the form of thin filaments was also observed in YBCO films deposited on vicinal cut substrates. ${ }^{41-44}$

\section{CONCLUSIONS}

Combining magneto-optical visualization of penetrated flux with magnetic ac susceptibility and dc magnetization measurements, we have investigated the early stages of flux penetration on $\mathrm{Nb}$ films with and without arrays of antidots. Our results show that ac susceptibility measurements can be used to detect vortex avalanches, either constructing $J_{c}(T)$ curves or monitoring the occurrence of a noisy behavior in Cole-Cole plots. We have also shown that, in the low-field regime, the roots of most dendrites are reused during the process of entrance and exit of flux, although some new dendrites and antidendrites might also be created at different points along the sample edges. From hysteresis loops measured at different temperatures, we were able to calculate both components of the ac susceptibility. MO images taken at several points of those loops enabled us to establish a reliable link among those three experimental techniques and, through this correspondence, visualize the flux distribution throughout the sample after an ac field cycle.

\section{ACKNOWLEDGMENTS}

This work was partially supported by the Methusalem Funding of the Flemish Government, the NES-ESF program, the Belgian IAP, the Fund for Scientific Research-Flanders (FWO-Vlaanderen), the UK Engineering and Physical Sciences Research Council, and the Brazilian funding agencies FAPESP and CNPq. A.V.S. is grateful for the support from the FWO-Vlaanderen. T.H.J. acknowledges the financial support of the Norwegian Research Council. W.A.O. thanks the Centre for Advanced Study (Norway) for the hospitality during the last stage of this work.

\footnotetext{
"Present address: Faculdade de Engenharia, UNESP - Universidade Estadual Paulista, Departamento de Física e Química, 15385-000 Ilha Solteira, SP, Brazil.

${ }^{1}$ C. P. Bean, Phys. Rev. Lett. 8, 250 (1962).

${ }^{2}$ C. P. Bean, Rev. Mod. Phys. 36, 31 (1964).

${ }^{3}$ Y. B. Kim, C. F. Hempstead, and A. R. Strnad, Phys. Rev. 129, 528 (1963).

${ }^{4}$ H. A. Ullmaier, Phys. Status Solidi 17, 631 (1966).

${ }^{5}$ J. R. Clem, J. Appl. Phys. 50, 3518 (1979).

${ }^{6}$ M. V. Indenbom, Th. Schuster, H. Kuhn, H. Kronmüller, T. W. Li, and A. A. Menovsky, Phys. Rev. B 51, 15484 (1995).

${ }^{7}$ A. V. Silhanek, J. Gutierrez, R. B. G. Kramer, G. W. Ataklti, J. Van de Vondel, V. V. Moshchalkov, and A. Sanchez, Phys. Rev. B 83, 024509 (2011).
}

${ }^{8}$ D. V. Denisov, D. V. Shantsev, Y. M. Galperin, Eun-Mi Choi, HyunSook Lee, Sung-Ik Lee, A. V. Bobyl, P. E. Goa, A. A. F. Olsen, and T. H. Johansen, Phys. Rev. Lett. 97, 077002 (2006).

${ }^{9}$ S. Hebert, L. Van Look, L. Weckhuysen, and V. V. Moshchalkov, Phys. Rev. B 67, 224510 (2003).

${ }^{10}$ F. Colauto, E. M. Choi, J. Y. Lee, S. I. Lee, V. V. Yurchenko, T. H. Johansen, and W. A. Ortiz, Supercond. Sci. Technol. 20, L48 (2007).

${ }^{11}$ F. Colauto, E. J. Patino, M. G. Blamire, and W. A. Ortiz, Supercond. Sci. Technol. 21, 045018 (2008).

${ }^{12}$ A. V. Silhanek, S. Raedts, and V. V. Moshchalkov, Phys. Rev. B 70, 144504 (2004).

${ }^{13}$ M. Menghini, R. J. Wijngaarden, A. V. Silhanek, S. Raedts, and V. V. Moshchalkov, Phys. Rev. B 71, 104506 (2005). 
${ }^{14}$ Matching effects are less pronounced at the temperature interval reported here (i.e., not very close to $T_{c}$ ). The commensurability field, characteristic of the lattice of ADs, is a reference for the window of magnetic fields at which the events treated here take place.

${ }^{15}$ V. V. Schmidt, The Physics of Superconductors, edited by P. Muller and A. V. Ustinov (Springer, Berlin, 1997).

${ }^{16}$ L. E. Helseth, R. W. Hansen, E. I. Il'yashenko, M. Baziljevich, and T. H. Johansen, Phys. Rev. B 64, 174406 (2001).

${ }^{17}$ The level of control of the zero-field condition was different for the 3 experimental setups employed: below $30 \mathrm{mOe}$ at the MPMS, below $250 \mathrm{mOe}$ at the MOI, and around 1 Oe at the PPMS.

${ }^{18}$ P. Leiderer, J. Boneberg, P. Brüll, V. Bujok, and S. Herminghaus, Phys. Rev. Lett. 71, 2646 (1993).

${ }^{19}$ C. A. Duran, P. L. Gammel, R. E. Miller, and D. J. Bishop, Phys. Rev. B 52, 75 (1995).

${ }^{20}$ T. H. Johansen, M. Baziljevich, D. V. Shantsev, P. E. Goa, Y. M. Galperin, W. N. Kang, H. J. Kim, E. M. Choi, M.-S. Kim, and S. I. Lee, Europhys. Lett. 59, 599 (2002).

${ }^{21}$ A. V. Bobyl, D. V. Shantsev, T. H. Johansen, W. N. Kang, H. J. Kim, E. M. Choi, and S. I. Lee, Appl. Phys. Lett. 80, 4588 (2002).

${ }^{22}$ I. A. Rudnev, S. V. Antonenko, D. V. Shantsev, T. H. Johansen, and A. E. Primenko, Cryogenics 43, 663 (2003).

${ }^{23}$ S. C. Wimbush, B. Holzapfel, and C. Jooss, J. Appl. Phys. 96, 3589 (2004).

${ }^{24}$ I. A. Rudnev, D. V. Shantsev, T. H. Johansen, and A. E. Primenko, Appl. Phys. Lett. 87, 042502 (2005).

${ }^{25}$ D. V. Denisov, A. L. Rakhmanov, D. V. Shantsev, Y. M. Galperin, and T. H. Johansen, Phys. Rev. B 73, 014512 (2006).

${ }^{26}$ M. Baziljevich, A. V. Bobyl, D. V. Shantsev, E. Altshuler, T. H. Johansen, and S. I. Lee, Physica C 369, 93 (2002).

${ }^{27}$ F. Colauto, E. Choi, J. Y. Lee, S. I. Lee, E. J. Patino, M. G. Blamire, T. H. Johansen, and W. A. Ortiz, Appl. Phys. Lett. 96, 092512 (2010).
${ }^{28}$ S. Kolesnik, V. Vlasko-Vlasov, U. Welp, G. W. Crabtree, T. Piotrowski, J. Wrobel, A. Klimov, P. Przystupski, T. Skoskiewicz, and B. Dabrowski, Physica C 341-348, 1093 (2000).

${ }^{29}$ V. Vlasko-Vlasov, U. Welp, V. Metlushko, and G. W. Crabtree, Physica C 341-348, 1281 (2000).

${ }^{30}$ E. H. Brandt and M. Indenbom, Phys. Rev. B 48, 12893 (1993).

${ }^{31}$ E. Zeldov, J. R. Clem, M. McElfresh, and M. Darwin, Phys. Rev. B 49, 9802 (1994).

${ }^{32}$ J. R. Clem and A. Sanchez, Phys. Rev. B 50, 9355 (1994).

${ }^{33}$ D.-X. Chen, C. Navau, N. Del-Valle, and A. Sanchez, Physica C 470, 89 (2010).

${ }^{34}$ V. V. Moshchalkov, M. Baert, V. V. Metlushko, E. Rosseel, M. J. Van Bael, K. Temst, Y. Bruynseraede, and R. Jonckheere, Phys. Rev. B 57, 3615 (1998).

${ }^{35}$ D. V. Shantsev, Y. M. Galperin, and T. H. Johansen, Phys. Rev. B 61, 9699 (2000).

${ }^{36}$ E. H. Brandt, Phys. Rev. B 55, 14513 (1997).

${ }^{37}$ E. H. Brandt, Phys. Rev. B 58, 6523 (1998).

${ }^{38}$ Notice that the same scale is used for the 3 magnetic loops shown. Further amplification of the $5 \mathrm{~K}$ loop would evidence some amount of hysteresis, due to penetration at the borders.

${ }^{39}$ R. B. Goldfarb and A. F. Clark, IEEE Trans. Magnetics, MAG-21, 332 (1985).

${ }^{40}$ M. S. Welling, R. J. Wijngaarden, C. M. Aegerter, R. Wördenweber, and P. Lahl, Physica C 404, 410 (2004).

${ }^{41}$ Ch. Jooss, R. Warthmann, and H. Kronmüller, Phys. Rev. B 61, 12433 (2000).

${ }^{42}$ A. Polyanskii, R. L. S. Emergo, J. Z. Wu, T. Aytug, D. K. Christen, G. K. Perkins, and D. Larbalestier, Phys. Rev. B 72, 174509 (2005).

${ }^{43}$ M. Djupmyr, G. Cristiani, H.-U. Habermeier, and J. Albrecht, Phys. Rev. B 72, 220507(R) (2005).

${ }^{44}$ V. V. Yurchenko, A. J. Qviller, P. B. Mozhaev, J. E. Mozhaeva, J. B. Hansen, C. S. Jacobsen, I. M. Kotelyanskii, A. V. Pan, and T. H. Johansen, Physica C 470, 799 (2010). 